\title{
Encoder Side Information Is Useful In Source Coding
}

\author{
Emin Martinian \\ Massachusetts Institute of Technology \\ Cambridge, MA USA \\ Email: emin@allegro.mit.edu
}

\author{
Gregory W. Wornell \\ Massachusetts Institute of Technology \\ Cambridge, MA USA \\ Email: gww@allegro.mit.edu
}

\author{
Ram Zamir \\ Tel Aviv University \\ Tel Aviv, Israel \\ Email: zamir@eng.tau.ac.il
}

\begin{abstract}
We introduce the idea of distortion side information, which does not directly depend on the source but instead affects the distortion measure. Such side information is not only useful at the encoder, but under many conditions of interest, knowing it at the encoder alone is sufficient and knowing it at the decoder alone is useless.
\end{abstract}

\section{INTRODUCTION}

We consider side information that is independent of the source but affects the distortion measure. Such distortion side information is a natural complement to the signal side information studied by Wyner and Ziv that depends on the source but does not affect the distortion measure. When both types of side information are considered, knowing the former only at the encoder and the latter only at the decoder is often asymptotically as good as complete knowledge of all side information. Potential examples of distortion side information include reliabilities for sensor readings and perceptual effects such as distortion masking, and sensitivity to context $[1,2]$.

\section{Problem Model}

We consider a source $\mathbf{x}=\left(x_{1}, x_{2}, \ldots, x_{n}\right)$, which is independent and identically distributed (i.i.d.) according to the distribution $p_{x}(x)$. The signal side information $\mathbf{w}$ is i.i.d. generated according to $p_{w \mid x}(w \mid x)$ and the distortion side information $\mathbf{q}$ is i.i.d., independent of $(\mathbf{x}, \mathbf{w})$, and generated according to $p_{q}(q)$. Distortion between a source $\mathbf{x}$ and reconstruction $\hat{\mathbf{x}}$ is measured via $(1 / n) \sum_{i=1}^{n} d\left(x_{i}, \hat{x}_{i} ; \boldsymbol{q}_{i}\right)$. Encoders, decoders, and rate-distortion functions are defined in the usual way.

As an example, consider a sensor application where each observation is observed at a different signal-to-noise ratio (SNR). We could let $q_{i}$ represent the SNR for observation $x_{i}$ and measure distortion via $d(x, \hat{x} ; q)=q \cdot(x-\hat{x})^{2}$. Intuitively, the more reliable observations where $q_{i}$ is large, should be communicated to the decoder more accurately than noisier observations where $q_{i}$ is small. If $\mathbf{q}$ is known by both encoder and decoder, a water-pouring distortion allocation achieves this goal. In the next section we analyze performance for distributed knowledge of $\mathbf{q}$.

\section{Main Results}

We denote the various possible rate-distortion functions by describing where the side-information is available and derive various relationships between them. For example, $R_{\mathrm{Q}-\mathrm{NONE}-\mathrm{W}-\mathrm{NONE}}(D)$ denotes the rate-distortion function without side information and $R_{\mathrm{Q}-\mathrm{NONE}-\mathrm{W}-\mathrm{DEC}}(D)$ denotes the rate-distortion function where $\mathbf{w}$ is available at the decoder studied by Wyner and Ziv [3]. Similarly, when all information is available at both encoder and decoder

This work has been supported in part by HP through the MIT/HP Alliance and by NSF under Grant No. CCR-0073520.
$R_{\mathrm{Q}-\mathrm{BOTH}-\mathrm{W}-\mathrm{BOTH}}(D)$ describes Csiszár and Körner's [4] generalization of Gray's conditional rate-distortion function $R_{\mathrm{Q}-\mathrm{NONE}-\mathrm{W}-\mathrm{BOTH}}(D)[5]$ to the case where the side information can affect the distortion measure.

We need various technical conditions corresponding to a "smooth" source and distortion measure; see $[1,6]$ for details. Our first result (generalizing [7]) is that having $\mathbf{q}$ only at the encoder and $\mathbf{w}$ only at the decoder is asymptotically optimal in high-resolution; see also $[1,2]$ for more general results.

Theorem 1 Let $\mathbf{q}$ be statistically independent of $(\mathbf{x}, \mathbf{w})$. For any source and scaled difference distortion measure $d(x, \hat{x} ; q)=d_{0}(q) \cdot d_{1}(x-\hat{x})$ satisfying the conditions in [1]

$$
\lim _{D \rightarrow 0} R_{\mathrm{Q}-\mathrm{ENC}-\mathrm{W} \text {-DEC }}(D)-R_{\mathrm{Q}-\mathrm{BOTH}-\mathrm{W} \text {-BOTH }}(D)=0 .
$$

Our next result shows when side information can be useless.

Theorem $\mathbf{2}$ Let $\mathbf{q}$ be statistically independent of $(\mathbf{x}, \mathbf{w})$ and consider a difference distortion measure of the form $d(x-\hat{x} ; q)$. Then $\mathbf{w}$ (respectively $\mathbf{q})$ provides no benefit when known only at the encoder (resp. decoder), i.e.,

$$
R_{\mathrm{Q}-\mathrm{DEC}-\mathrm{W}-\mathrm{ENC}}(D)=R_{\mathrm{Q}-\mathrm{NONE}-\mathrm{W}-\mathrm{NONE}}(D) .
$$

Furthermore, in [1] we generalize these results to show that the value of a given type of side information at the encoder/decoder is essentially independent of where the other type of side information is known. For example, under the conditions of the above theorems we derive results like $R_{\mathrm{Q} \text {-ENC-W-ENC }}(D)=R_{\mathrm{Q}-\mathrm{ENC}-\mathrm{W} \text {-NONE }}(D)$, $R_{\mathrm{Q}-\mathrm{DEC}-\mathrm{W}-\mathrm{DEC}}(D)=R_{\mathrm{Q}-\mathrm{NONE}-\mathrm{W}-\mathrm{DEC}}(D)$, and

$$
\lim _{D \rightarrow 0} R_{\mathrm{Q}-\mathrm{ENC}-\mathrm{W}-\mathrm{ENC}}(D)-R_{\mathrm{Q}-\mathrm{BOTH}-\mathrm{W}-\mathrm{ENC}}(D)=0 .
$$

Thus knowing distortion side information, q, at the encoder and signal side information, $\mathbf{w}$, at the decoder is asymptotically optimal in a wide variety of scenarios.

\section{REFERENCES}

[1] E. Martinian, G. W. Wornell, and R. Zamir, "Source coding encoder side information." in preparation.

[2] E. Martinian, G. W. Wornell, and R. Zamir, "Source coding with distortion side information at the encoder," in Proc. Data Compression Conference, pp. 172-181, March 2004.

[3] A. D. Wyner and J. Ziv, "The rate-distortion function for source coding with side information at the decoder," IEEE Trans. Inform. Theory, vol. 22, pp. 1-10, Jan. 1976.

[4] I. Csiszár and J. Körner, Information Theory: Coding Theorems For Discrete Memoryless Systems. Academic Press, 1981.

[5] R. Gray, "A new class of lower bounds to information rates of stationary sources via conditional rate-distortion functions," IEEE Trans. Inform. Theory, vol. 19, pp. 480-489, July 1973.

[6] T. Linder, R. Zamir, and K. Zeger, "On source coding with side-information-dependent distortion measures," IEEE Trans. Inform. Theory, vol. 46, pp. 2697-2704, Nov. 2000.

[7] R. Zamir, "The rate loss in the Wyner-Ziv problem," IEEE Trans. Inform. Theory, vol. 42, pp. 2073-2084, Nov. 1996. 